\title{
VISIÓN HOLÍSTICA DE LAS TECNOLOGÍAS DE MÉTODOS Y TÉCNICAS DIDÁCTICAS EN EL PROCESO ENSEÑANZA APRENDIZAJE EN LA EDUCACIÓN SUPERIOR
}

\section{Holistic Vision Technologies Methods and teaching techniques in the teaching-learning process in Higher Education}

Amparo Bienvenida Baque Morán ${ }^{1}$ Miguel Ángel Jaime Baque ${ }^{2}$ Carlos Artemidoro Zea Barahona ${ }^{3}$ Gino Iván Ayón Ponce ${ }^{4}$

1. Licenciada en Ciencias de la Educación especialización Comercio y Administración, Magister en Docencia Universitaria e Investigación Educativa, Docente, Carrera Gestión Empresarial, Facultad de Ciencias Económicas Universidad Estatal del Sur de Manabí, Ecuador. E-mail: ambamo@hotmail.es

2. Ingeniero en Comercio Exterior, Ingeniero en Medio Ambiente, Magister en Contabilidad y Auditoría, Docente, Carrera Gestión Empresarial, Facultad de Ciencias Económicas Universidad Estatal del Sur de Manabí, Ecuador. E-mail: angel.m.86@hotmail.com

3. Economista, Magister en Gerencia e Investigación Educativa, Docente, Carrera Gestión Empresarial, Facultad de Ciencias Económicas Universidad Estatal del Sur de Manabí, Ecuador. E-mail: caryfran3@hotmail.com

4. Ingeniero Comercial, Magister en Gerencia Educativa, Docente, Carrera Gestión Empresarial, Facultad de Ciencias Económicas Universidad Estatal del Sur de Manabí, Ecuador. E-mail: ginoayon1@hotmail.com

\section{Citación sugerida:}

Baque Morán, A.B., Jaime Baque, M.Á., Zea Barahona, C.A. y Ayón Ponce, G.I. (2016). 3C Empresa: innovación y pensamiento crítico, 5(3) 63-85. DOI: <http://dx.doi.org/10.17993/3cemp.2016.050327.63-85/>. 


\section{RESUMEN}

Este artículo refleja una perspectiva sobre el trabajo docente, que está orientado al cambio de la acción educativa para responder a las necesidades individuales de los estudiantes y las necesidades de la sociedad. El objetivo consistió en analizar una visión holística de métodos y técnicas didácticas en el proceso enseñanza aprendizaje de los estudiantes de la carrera de Ingeniería en Computación y Redes de la Universidad Estatal del Sur de Manabí, en base a una guía metodológica de procesos didácticos holísticos. La metodología utilizada fue a través de instrumentos valorativos basados en documentos de referencias que ayudaron a justificar esta problemática, este estudio generó resultados altamente positivos para los principales protagonistas de la investigación: docentes y alumnos. Se concluyó que los métodos y técnicas son recursos didácticos indispensables que potencian el trabajo docente y les permite a los catedráticos mejorar su práctica pedagógica, al actuar como mediadores de aprendizajes significativos a través del uso de una metodología constructivista, a los estudiantes la oportunidad de innovar y construir sus propios aprendizajes, a fin de afianzar los conocimientos prácticos teóricos en base a la aplicación de la propuesta "Guía Metodológica de Procesos Didácticos holísticos".

\section{ABSTRACT}

This article reflects a perspective on teaching work, which is oriented to the change of educational activities to meet the individual needs of students and the needs of society. The aim was to analyze a holistic view of teaching methods and techniques in the teachinglearning process of the students studying Computer Engineering and Networks Southern State University of Manabí, based on a methodological guide learning processes holistic, The methodology used was through evaluative instruments based on reference documents that helped justify this problem, this study generated highly positive for the main protagonists of research results: teachers and students. It was concluded that the methods and techniques are indispensable teaching resources enhance the teaching work and allows professors to improve their teaching practice, to act as mediators of significant learning through the use of a constructivist methodology; students the opportunity to innovate and build their own learning, to strengthen the skills based on the theoretical application of the proposed methodological guide to learning processes holistic.

\section{PALABRAS CLAVE}

Holística; métodos; técnicas; enseñanza aprendizaje; procesos didácticos

\section{KEYWORDS}

Holistic; methods; techniques; teaching and learning; learning processes 


\section{INTRODUCCION}

El Espacio Ecuatoriano de Educación Superior está realizando un cambio drástico en varias dimensiones del trabajo docente: en la planificación, en la enseñanza, en la evaluación, en la tutoría, entre otros parámetros. Estos nuevos desafíos están requiriendo que los profesores hagan una readaptación de sus prácticas educativas, conocimientos y creencias para adecuarse a los nuevos ambientes de aprendizaje.

Hoy en día, la educación exige cambios en el sistema educativo, donde el docente debe estar preparado para enfrentar la diversidad del educando. Esto requiere que el profesor desarrolle ciertas habilidades fuera del contexto del plan tradicional de estudios, conociendo las formas de enseñanza holística activa y práctica que más se adapten a su realidad.

Según Gallegos, (2005), menciona que en forma global los métodos de enseñanza holística por lo general incorporan enfoques primordiales que abarca todo el cerebro, auxiliándose de las inteligencias múltiples (lingüística, musical, lógica matemática, espacial, corporalkinestésica, interpersonal, intrapersonal, tecnológica y naturalista). En el aprendizaje de toda la estructura del cerebro, el tema que hay que aprender es abordado desde múltiples perspectivas, en especial a aquellos que incluyen a tantos sentidos como sea posible. El objetivo es permitir que la experiencia del estudiante en cualquier área o sub-área sea a nivel sensorial, emocional e intelectual para que pueda edificar una red neuronal relacionada con la temática (p.14).

Por su parte, García, Trujillo y Pérez, (2010), presentaron una propuesta, basada en experiencias de carácter metodológico aplicadas durante cinco cursos, sobre la utilización de métodos productivos en el proceso de enseñanza aprendizaje. Los métodos fueron aplicados en tareas docentes planificadas en todos los temas del programa y en la última evaluación de la asignatura, utilizando la modalidad de juegos con diferentes variantes, con la aplicación de estos método lograron incrementar el estudio independiente que contribuyó a: mejorar la expresión oral, desarrollar la imaginación, buscar alternativas para la resolución de un problema dado y favorecer los cambios de conducta mediante el intercambio grupal.

En este orden de ideas, Montes de Oca y Machado Ramírez, (2011), sostienen en su artículo que la Didáctica centrada en el estudiante que exige la utilización de estrategias y métodos adecuados, en los que el aprendizaje se conciba cada vez más como resultado del vínculo entre lo afectivo, lo cognitivo, las interacciones sociales y la comunicación. Este artículo ofrece elementos teóricos esenciales que permiten asumir posiciones fundamentadas acerca de las estrategias docentes y los métodos de enseñanza-aprendizaje, lo que en ocasiones se aborda desde diversas posiciones, no siempre consistentes y con múltiples acepciones e interpretaciones.

Por otra parte, Vera, Osses, y Schiefelbein (2012), señalan en su investigación sobre las creencias en el proceso de enseñanza-aprendizaje se han convertido en el último tiempo en un tema de gran preocupación, debido a la creciente evidencia que señala que las creencias 
tienen un fuerte impacto en el quehacer pedagógico de los profesores. En este artículo se desarrolla una visión que le permitiría tener una visión hipotética, desde el estudio de las creencias, una de las posibles variables personales del profesor para explicar los resultados de aprendizaje en la educación. Estos autores concluyen la necesidad de investigar las creencias que sustentan los profesores rurales respecto del proceso educativo, dada su relevancia en el proceso de enseñanza y aprendizaje de los estudiantes.

En la investigación descrita por De León C., (2013), al hacer referencia a Formación Docente Universitaria. Estilos Pedagógicos de Enseñanza y Gestión del Conocimiento, es posible reportar que, progresivamente, estos han ido alcanzando una presencia e interrelación creciente en el ámbito educativo. Dichos términos en gran medida orientan, dirigen la labor docente en interacción con otros actores del hecho instruccional, con la intencionalidad de desarrollar de manera efectiva dicha labor.

Otra investigación relacionada con uno de los tópicos abordados y descritos en un artículo es la preparación de los docentes para dirigir el proceso de enseñanza aprendizaje utilizando niveles de asimilación, Sierra, Castellano, y García, (2013), parten de un trabajo investigativo en la educación superior que está encaminado a la solución teórico práctica de sus problemas, partiendo de esta premisa los autores se proponen como objetivo caracterizar el nivel de preparación de los docentes para dirigir el proceso de enseñanza aprendizaje utilizando los niveles de asimilación.

Teniendo en cuenta lo antes expuesto sobre estudios que tiene similitud con el tema de investigación, donde se delineó como objetivo la aplicación de una visión holística de métodos y técnicas didácticas en el proceso enseñanza aprendizaje de los estudiantes de la carrera de Ingeniería en Computación y Redes de la Universidad Estatal del Sur de Manabí, en base a una Guía Metodológica de Procesos Didácticos, (Christian, 2016) expresa que la relación educación, aprendizaje e innovación forman un papel preponderante para el desarrollo de la catedra, ampliando la brecha de vínculo con la comunidad para alcanzar un desarrollo sustentable y sostenible a través de proyectos tecnológicos.

\section{MATERIALES Y MÉTODOS}

El marco metodológico de investigación fue cualitativa, ya que permitió observar de una forma estructurada la realidad en estudio por medio de técnicas e instrumentos (encuestas y entrevistas) que se aplicaron a la coordinadora de carrera, decano de facultad, docentes y estudiantes con los cuales se recopiló la información para caracterizar la investigación del trabajo docente y su relación con el desarrollo del proceso enseñanza aprendizaje; a su vez los resultados obtenidos, permitieron elaborar la propuesta de solución del problema en cuestión, permitiendo concatenar al investigador con los sujetos involucrados; y cuantitativa, ya que se pudo agrupar elementos estadísticos sobre las variables y resultados. De igual manera, se utilizaron diferentes fuentes bibliográficas, lo cual permitió indagar algunos fundamentos teóricos- metodológicos - científicos para determinar la teoría sobre el análisis de la visión holística de métodos y técnicas didácticas en el proceso 
enseñanza aprendizaje de los estudiantes de la carrera de Ingeniería en Computación y Redes de la Universidad Estatal del Sur de Manabí, con el fin de seleccionar datos, para la demostración de hipótesis y construcción de lineamientos relevantes. Los métodos utilizados en la presente investigación fueron el método científico, hermenéutico, analítico sintético, hipotético deductivo y estadístico.

Para la tabulación y análisis estadístico se utilizó el paquete de Microsoft Office 2013, donde se extrajeron resultados muy valiosos para diseñar la propuesta que se enmarcó en la implementación de un manual como guía metodológica de procesos didácticos para el trabajo docente en la carrera de Ingeniería en Computación y Redes, cuyo objeto es el perfeccionamiento del pensamiento, la creatividad y la producción de aprendizajes significativos.

Tabla 1. Preguntas de investigación y técnicas utilizadas.

\section{PREGUNTAS DE INVESTIGACIÓN}

¿Cuáles son los procesos teóricosmetodológicos que orientan el trabajo docente y su relación con el desarrollo del proceso enseñanza-aprendizaje de los alumnos de la Carrera de Ingeniería en Computación y Redes de la Universidad Estatal del Sur de Manabí?

¿Cómo se manifiesta la relación entre la operatividad de los métodos, técnicas y procedimientos y el desarrollo del proceso enseñanza - aprendizaje de los alumnos de la Carrera de Ingeniería en Computación y Redes de la Universidad Estatal del Sur de Manabí?

\section{TÉCNICAS UTILIZADAS}

Entrevista, aplicada a autoridades de la carrera para complementar el análisis del trabajo docente y su relación con el desarrollo del proceso enseñanza aprendizaje.

Encuesta a docentes y a una muestra representativa de estudiantes, para analizar el trabajo docente y su relación con el desarrollo del proceso enseñanza aprendizaje.

Observación y Encuesta a docentes y a una muestra representativa de estudiantes.

Encuesta dirigida a docentes y estudiantes de la carrera, para analizar si el trabajo docente aplicado en la carrera tiene relación con el proceso enseñanza aprendizaje. 
MUESTRA ESTRATIFICADA CON AFIJACIÓN UNIFORME:

Tabla 2. cuadro de muestra probabilística estratificada.

\begin{tabular}{|c|c|c|c|}
\hline $\begin{array}{l}\text { ESTRATO POR } \\
\text { CATEGORÍAS }\end{array}$ & $\begin{array}{l}\text { PARALELOS POR SEMESTRE Y } \\
\text { SECCIÓN }\end{array}$ & $\begin{array}{c}\text { TOTAL } \\
\text { POBLACIÓN } \\
\text { (fh) = } 0.6736 \\
\text { Nh(fn) }=n h\end{array}$ & $\begin{array}{l}\text { MUESTRA } \\
\text { (nh) }\end{array}$ \\
\hline 1 & Primero Paralelo "A" Matutina & 21 & 14 \\
\hline 2 & Primero Paralelo "B" Nocturno & 24 & 16 \\
\hline 3 & Segundo Paralelo "A" Matutina & 18 & 12 \\
\hline 4 & Segundo Paralelo "B" Nocturno & 20 & 13 \\
\hline 5 & Tercero Paralelo "A" Matutina & 14 & 9 \\
\hline 6 & Tercero Paralelo "B" Nocturno & 17 & 12 \\
\hline 7 & Cuarto Paralelo "A" Matutino & 13 & 9 \\
\hline 8 & Cuarto Paralelo "B" Nocturno & 18 & 12 \\
\hline 9 & Quinto Paralelo "A" Nocturno & 22 & 15 \\
\hline 10 & Séptimo Paralelo "A" Nocturno & 17 & 12 \\
\hline 11 & Décimo Paralelo "A" Nocturno & 9 & 6 \\
\hline \multicolumn{2}{|l|}{ TOTAL } & 193 & 130 \\
\hline
\end{tabular}

\section{RESULTADOS Y DISCUSIÓN}

A partir de los resultados de la encuesta y entrevista, se realizó la contextualización del diagnóstico de la IES, específicamente la carrera Ingeniería en Computación y Redes para establecer los factores críticos en los procesos teóricos-metodológicos que orientan el trabajo docente y su relación con el desarrollo del proceso enseñanza-aprendizaje.

\section{1. ¿Cuál es su formación académica?}

Tabla 3. Formación académica.

\begin{tabular}{|l|c|c|}
\hline RESPUESTAS & DOCENTES & \\
\hline Analistas en Sistema & Frecuencia & $\%$ \\
\hline Ingenieros Eléctricos & 6 & 35 \\
\hline Ingenieros Civiles & 3 & 18 \\
\hline Licenciados en CC.EE. & 5 & 18 \\
\hline \hline TOTAL & 17 & 29 \\
\hline \multicolumn{2}{|c|}{ Fuente: Encuestas aplicadas a docentes. } \\
Elaboración: Equipo de investigación.
\end{tabular}




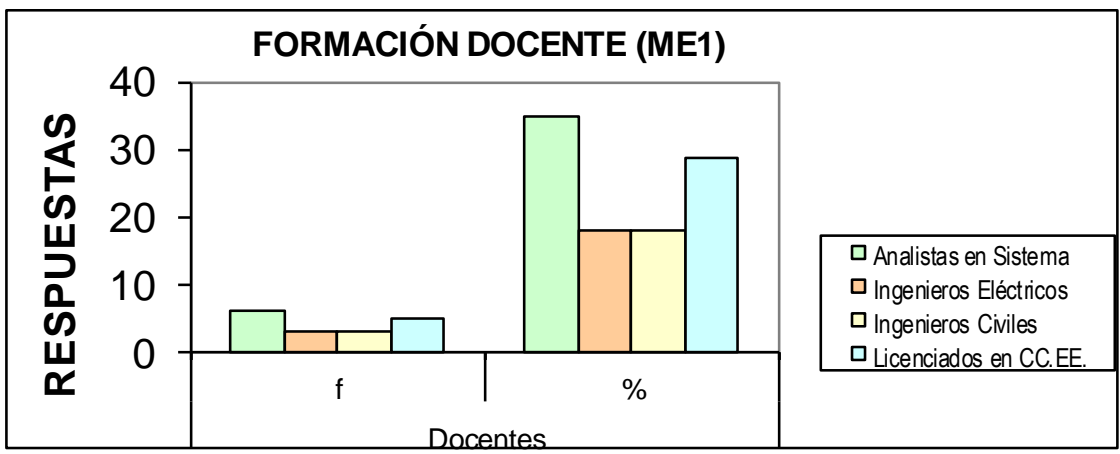

Gráfico 1: Formación docente.

De acuerdo a los resultados obtenidos en la encuesta, el 35\% de docentes poseen el título de Analistas en Sistemas, el $29 \%$ son Licenciados en Ciencias de la Educación y el $18 \%$ Ingenieros Eléctricos y Civiles.

Es evidente que, el mayor porcentaje de docentes NO posee formación pedagógica, lo que hace que exista debilidad en el proceso enseñanza aprendizaje. Por lo tanto, se hace necesario la aplicación de una metodología eficiente, ya que es el pilar fundamental del trabajo docente y su relación con el desarrollo del proceso enseñanza aprendizaje.

\section{2. ¿Qué tiempo tiene usted ejerciendo docencia universitaria?}

Tabla 4. Tiempo de ejercicio como docente.

\begin{tabular}{|c|c|c|}
\hline \multirow[t]{2}{*}{ RESPUESTAS } & \multicolumn{2}{|c|}{ DOCENTES } \\
\hline & FRECUENCIA & $\%$ \\
\hline 1 - 3 AÑOS & 8 & 47 \\
\hline $4-7$ AÑOS & 8 & 47 \\
\hline 8 - 11 AÑOS & 1 & 6 \\
\hline 12 - 15 AÑOS & 0 & 0 \\
\hline TOTAL & 17 & 100 \\
\hline
\end{tabular}

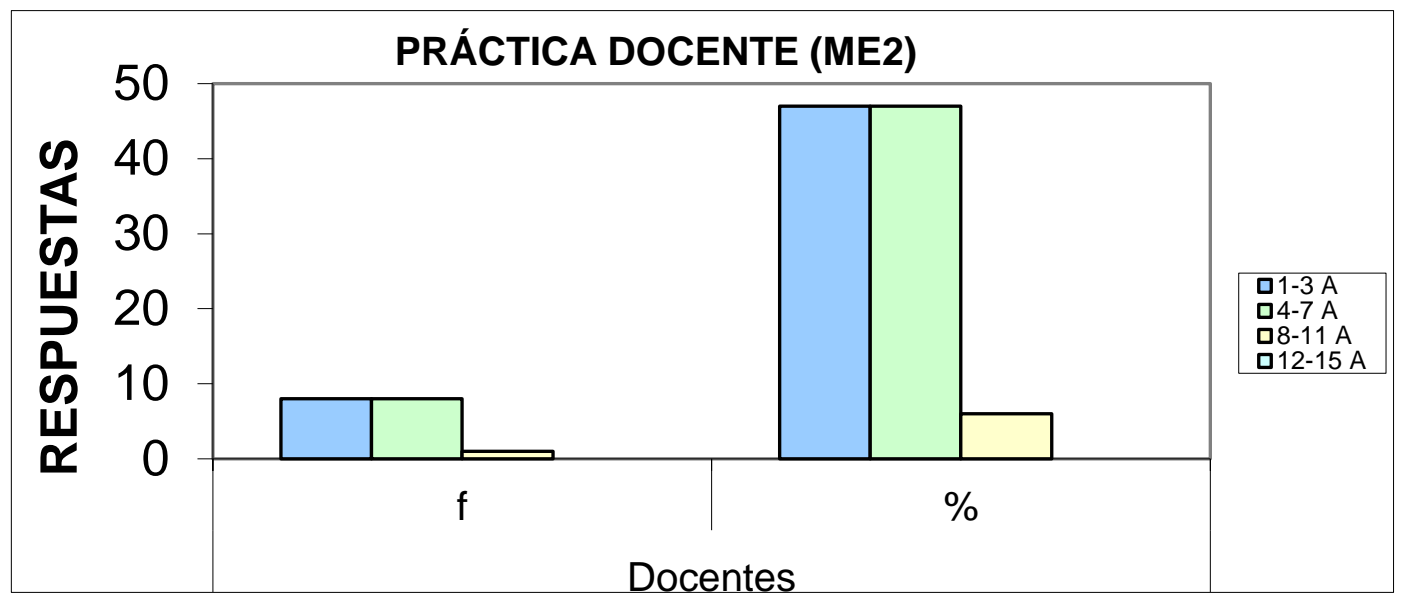

Gráfico 2: Práctica docente. 
El $47 \%$ de docentes tienen en la práctica docente de 1 a 3 años y el mismo porcentaje de 4 a 7 años; el $6 \%$ de 8 a 11 años.

Lo que se puede notar que en su mayoría los docentes tienen poca experiencia en docencia. Esto incide directamente en el aprendizaje de los profesionales en formación, puesto que el docente debe de estar preparado para enfrentar el desafío de competitividad que exige la sociedad.

De acuerdo con lo anterior se puede afirmar que la labor docente no se basa simplemente en exponer una materia, sino que se requiere de conocimientos pedagógicos y bases didácticas, pues la didáctica contribuye a dominar con eficacia la acción del docente y al mismo tiempo incrementar el interés del alumno.

3. ¿Cuál es la relación docente- estudiante?

Tabla 5. Relación docente-estudiante.

\begin{tabular}{|l|c|c|c|c|}
\hline & RESPUESTAS & \multicolumn{2}{c}{ DOCENTES } & \multicolumn{2}{c|}{ ESTUDIANTES } \\
\hline HORIZONTAL & 5 & 29 & 40 & $\mathbf{3 1}$ \\
\hline VERTICAL & 12 & 71 & 90 & $\mathbf{6 9}$ \\
\hline TOTAL & $\mathbf{1 7}$ & $\mathbf{1 0 0}$ & $\mathbf{1 3 0}$ & $\mathbf{1 0 0}$ \\
\hline
\end{tabular}

Fuente: Encuestas aplicadas a docentes, directivo y estudiantes.

Elaboración: Equipo de investigación.

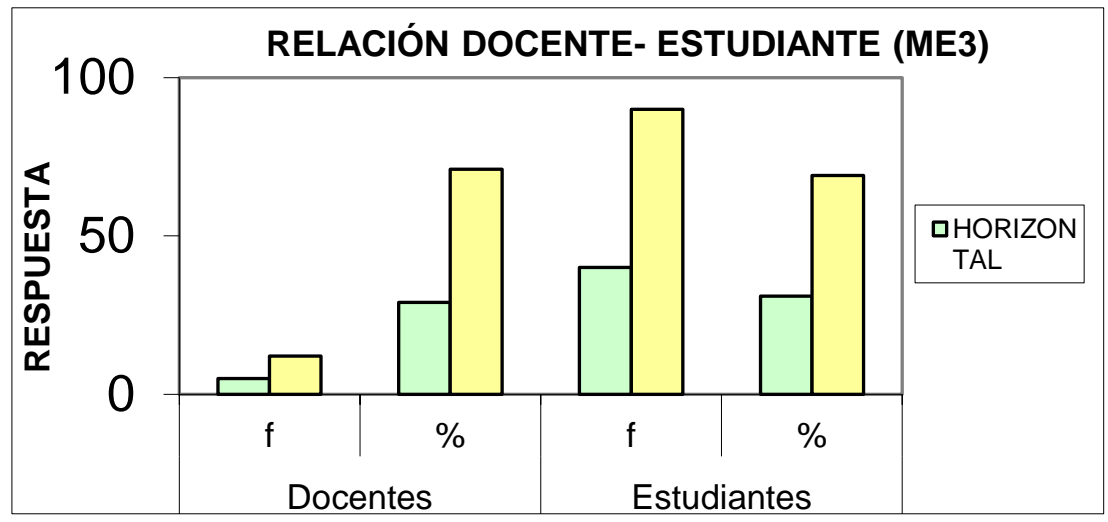

Gráfico 3. Relación entre docente y estudiante.

El $71 \%$ de docentes y el $69 \%$ de estudiantes señalan que la relación docente-estudiantes es vertical, el $29 \%$ de docentes y $31 \%$ de estudiantes indicaron que la relación es horizontal. Analizando los porcentajes se puede determinar que los docentes son los que educan, disciplinan, hablan y él es el sujeto de la clase, él sabe. En cambio el alumno es el educado, es disciplinado, solo escucha, sigue la prescripción del maestro recibe el programa en forma de "depósito" el alumno es el objeto del proceso.

\section{4.¿QUÉ MODELOS PEDAGÓGICOS CONSIDERA USTED EN EL DESARROLLO DEL PROCESO ENSEÑANZA APRENDIZAJE?}

Tabla 6. Modelos pedagógicos en el proceso de enseñanza aprendizaje.

\begin{tabular}{|l|c|c|c|c|}
\hline \multicolumn{1}{|c}{ RESPUESTAS } & \multicolumn{3}{c}{ DOCENTES } & \multicolumn{2}{c|}{ ESTUDIANTES } \\
\hline TRADICIONAL & 2 & 12 & 108 & $\mathbf{8 3}$ \\
\hline TECNOCRÁTICO & 2 & 12 & 0 & $\mathbf{0}$ \\
\hline
\end{tabular}




\begin{tabular}{|l|c|c|c|c|}
\hline CRÍTICO & 5 & 29 & 5 & $\mathbf{4}$ \\
\hline EXPERIENCIAL & 3 & 18 & 8 & $\mathbf{6}$ \\
\hline POTENCIADOR & 5 & 29 & 9 & $\mathbf{7}$ \\
\hline TOTAL & $\mathbf{1 7}$ & $\mathbf{1 0 0}$ & $\mathbf{1 3 0}$ & $\mathbf{1 0 0}$ \\
\hline
\end{tabular}

Fuente: Encuestas aplicadas a docentes, directivo y estudiantes.

Elaboración: Equipo de investigación.

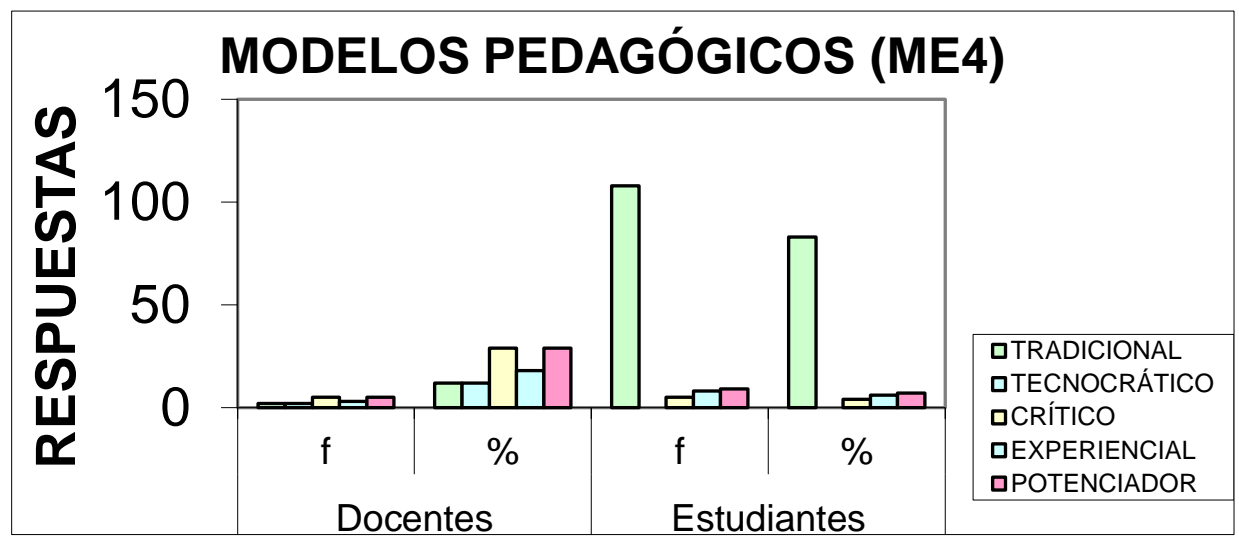

Gráfico 4. Modelos pedagógicos en la enseñanza.

El $29 \%$ de docentes utilizan modelos pedagógicos Crítico y Potenciador; y, los estudiantes indican que el $83 \%$ de los profesores son tradicionalistas. Como se puede notar en la gráfica existe una contradicción en las dos posiciones.

Con los resultados expuestos se puede deducir que los docentes de la carrera siguen trabajando con el modelo tradicional, donde tienen un sistema rígido de autoridad; quien tiene la mayor jerarquía es quien toma las decisiones, y el alumno que es el que está al final de esta cadena autoritaria, carece de poder.

\section{5.¿Qué metodología utiliza usted en el proceso enseñanza aprendizaje?}

Tabla 7. Métodos en el proceso de enseñanza aprendizaje.

\begin{tabular}{|l|c|c|c|c|}
\hline \multirow{2}{*}{ RESPUESTAS } & \multicolumn{2}{c}{ DOCENTES } & \multicolumn{2}{c|}{ ESTUDIANTES } \\
\hline & FRECUENCIA & $\mathbf{\%}$ & FRECUENCIA & $\%$ \\
\hline INVESTIGATIVO & $\mathbf{1 2}$ & $\mathbf{7 1}$ & $\mathbf{1 5}$ & 12 \\
\hline TRANSMITIVO & $\mathbf{0}$ & $\mathbf{0}$ & $\mathbf{9 5}$ & $\mathbf{7 3}$ \\
\hline ORGANIZATIVO & $\mathbf{5}$ & $\mathbf{2 9}$ & $\mathbf{2 0}$ & $\mathbf{1 5}$ \\
\hline \hline TOTAL & $\mathbf{1 7}$ & $\mathbf{1 0 0}$ & $\mathbf{1 3 0}$ & $\mathbf{1 0 0}$ \\
\hline
\end{tabular}

Fuente: Encuestas aplicadas a docentes, directivo y estudiantes.

Elaboración: Equipo de investigación. 


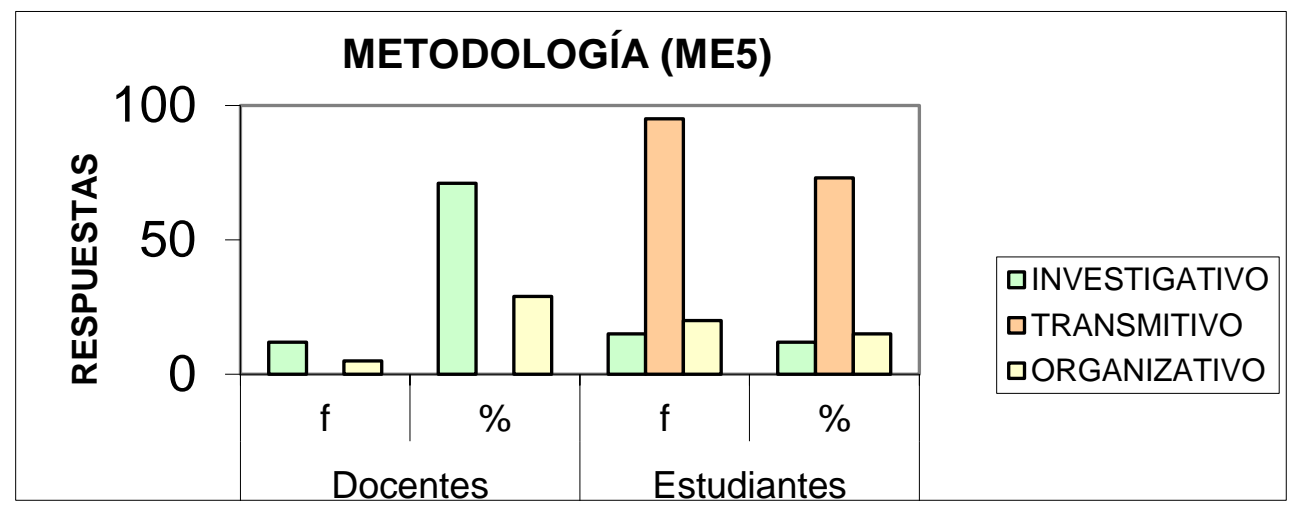

Gráfico 5. Metodología en el proceso de enseñanza.

El $71 \%$ de docentes manifiestan que el método que utilizan es el investigativo; y los estudiantes el $73 \%$ indican que los profesores aplican métodos de transmisión, el $29 \%$ de docentes trabajan con el método organizativo y el $15 \%$ de estudiantes afirman que los profesores emplean el mismo método.

Demostrando con ello, que existe una deficiente utilización de métodos, es necesario que se establezcan tendencias actuales en el mundo educativo concibiendo con una metodología para organizar mejor los sistemas de actividades, comunicación y motivación. La metodología del trabajo docente/ y los alumnos, es uno de los puntos cruciales y porque no decirlo el punto clave que enmarca las relaciones entre todos los sujetos que conforman el proceso enseñanza aprendizaje. El uso de una determinada metodología, o distintas estrategias metodológicas puede hacer óptimo, por un lado el proceso de enseñanza del docente, por otro el proceso de aprendizaje de los alumnos. El profesor tiene un papel crítico en la creación de un clima de relaciones en el aula que trasforme a ésta en un lugar de trabajo compartido.

6. Durante el proceso de aprendizaje considera frecuentemente:

Tabla 8. Tipos de situaciones en el proceso de aprendizaje.

\begin{tabular}{|c|c|c|c|c|}
\hline \multirow[t]{2}{*}{ RESPUESTAS } & \multicolumn{2}{|c|}{ DOCENTES } & \multicolumn{2}{|c|}{ ESTUDIANTES } \\
\hline & FRE & & FREC & $\%$ \\
\hline DISCUSIONES & 10 & 59 & 90 & 69 \\
\hline SITUACIONES PROBLÉMICAS & 2 & 12 & 15 & 12 \\
\hline ESTUDIO DIRIGIDO & 5 & 29 & 25 & 19 \\
\hline CONFERENCIA MAGISTRAL & 0 & 0 & 0 & 0 \\
\hline TOTAL & 17 & 100 & 130 & 100 \\
\hline
\end{tabular}




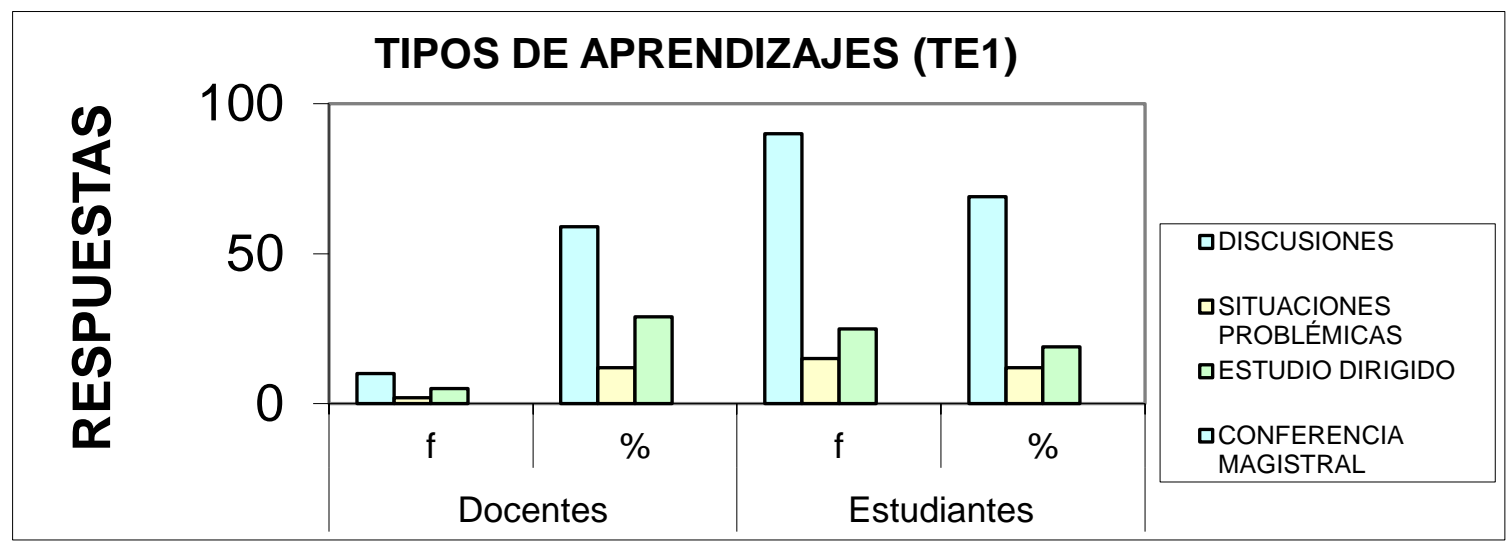

Gráfico 6. Tipos de aprendizaje.

El 59\% de los docentes considera la discusión en el desarrollo de la clase; y el $69 \%$ de los estudiantes coinciden con la opinión de los profesores. De la misma manera el $29 \%$ de los docentes y el $19 \%$ de los estudiantes indican que también se da el estudio dirigido.

En vista de las alternativas anteriormente planteadas es necesario que se estructuren mecanismos de estrategias para afianzar conocimientos en el proceso enseñanzaaprendizaje, en base a la aplicación de situaciones problémicas entremezcladas con otras actividades escolares y así favorecer el aprendizaje creativo.

En el proceso de enseñanza aprendizaje se debe estimular el empleo de procedimiento que les propicie a los alumnos el logro de la asimilación productiva de los conocimientos profesionales.

Es necesario utilizar técnicas que se inserten en un procedimiento metodológico generalizado para la solución de situaciones problémicas profesionales.

Ahora bien, para utilizar adecuadamente las técnicas y procedimientos metodológicos generalizados y que se produzca la asimilación productiva de los conocimientos es necesario, además, "crear las condiciones necesarias que promuevan e intensifiquen la motivación, a través de la creación de significados para los estudiantes". (Fuentes, 1998; 155).

\section{7. ¿Qué técnicas de aprendizaje utiliza con mayor frecuencia?}

Tabla 9. Técnicas de aprendizaje.

\begin{tabular}{|c|c|c|c|c|}
\hline RESPUESTAS & \multicolumn{2}{|c|}{ DOCENTES } & \multicolumn{3}{|c|}{ ESTUDIANTES } \\
\hline & FRECUENCIA & $\%$ & FRECUENCIA & $\mathbf{\%}$ \\
\hline EXPOSICIÓN & 4 & 24 & 80 & $\mathbf{6 2}$ \\
\hline LLUVIA DE IDEAS & 3 & 17 & 13 & $\mathbf{1 0}$ \\
\hline TECNICAS VISUALES & 3 & 17 & 10 & $\mathbf{8}$ \\
\hline OBSERVACIÓN & 2 & 12 & 6 & $\mathbf{5}$ \\
\hline MESA REDONDA & 2 & 12 & 4 & $\mathbf{3}$ \\
\hline TALLERES & 1 & 6 & 4 & $\mathbf{3}$ \\
\hline LECTURA COMENTADA & 1 & 6 & 4 & $\mathbf{3}$ \\
\hline OTRAS & 1 & 6 & 9 & $\mathbf{6}$ \\
\hline \hline TOTAL & $\mathbf{1 7}$ & $\mathbf{1 0 0}$ & $\mathbf{1 3 0}$ & $\mathbf{1 0 0}$ \\
\hline
\end{tabular}

Fuente: Encuestas aplicadas a docentes, directivo y estudiantes.

Elaboración: Equipo de investigación. 


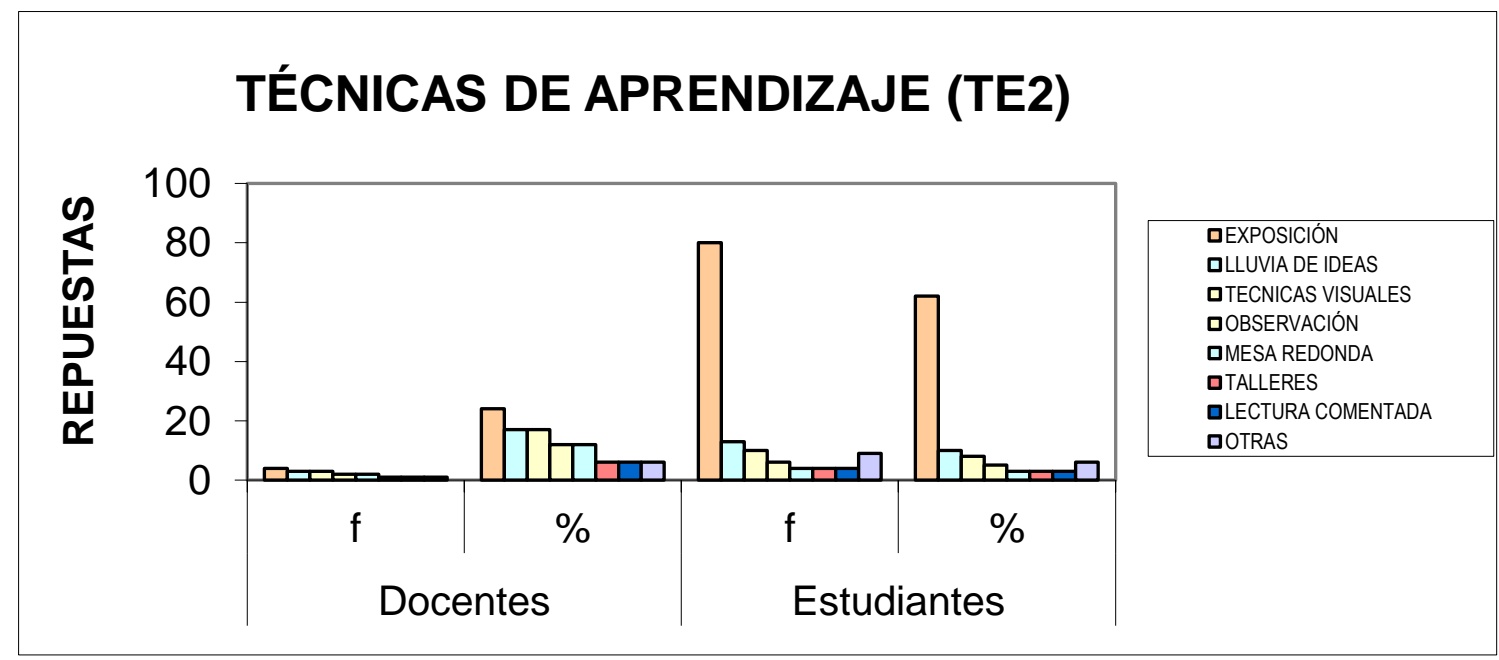

Gráfico 7. Técnicas de aprendizaje.

Las técnicas que utilizan con mayor frecuencia los docentes de la Carrera de Ingeniería en Computación y Redes son las exposiciones con el $24 \%$, lluvias de ideas y técnicas visuales con el $17 \%$, los estudiantes indican que el docente más utiliza la exposición con un $62 \%$, y el $10 \%$ lluvia de ideas.

De esto se puede deducir que los docentes están aplicando muy pocas técnicas que puedan fortalecer en un sistema amplio el proceso de enseñanza-aprendizaje, de tal manera que el alumno sea más dinámico y creativo. La metodología que utilizan los docentes es la clásica, porque a veces se interrumpe la exposición con dinámicas, trabajo en grupos, pequeñas investigaciones, para después volver a la exposición del docente, es decir que en la práctica docente, los estudiantes no aprenden actividades y valores a relacionarse con sus compañeros, a investigar, entre otras.

Con los resultados expuestos se puede deducir que los docentes de la carrera siguen trabajando con el modelo tradicional, donde tienen un sistema rígido de autoridad; quien tiene la mayor jerarquía es quien toma las decisiones, y el alumno que es el que está al final de esta cadena autoritaria, carece de poder. La ilustración 1 demuestra los resultados de cómo es la educación y que cambios se deben realizar. 


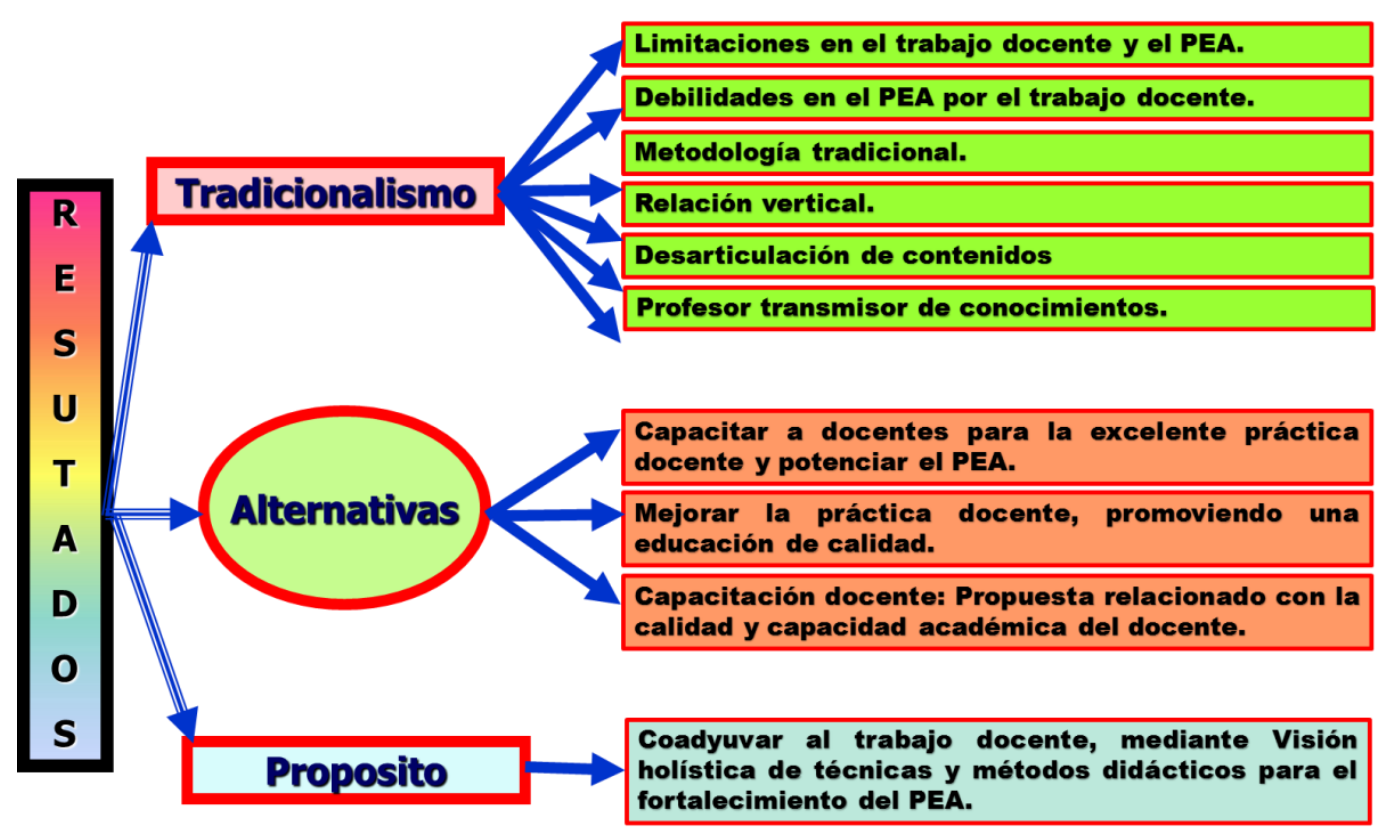

Ilustración 1. La educación tradicional y la holística de la educación.

Elaborado por: Investigadores.

Por lo tanto, con la aplicación de la enseñanza holística, de métodos y técnicas en la enseñanza aprendizaje en la Educación Superior, se cambia el trabajo docente educativo con tendencias actuales y concibiendo la creatividad con una metodología activa que enmarca las relaciones entre todos los sujetos, por un lado el proceso de enseñanza del docente, por otro el proceso de aprendizaje de los alumnos. El profesor tiene un papel crítico en la creación de un clima de relaciones en el aula que trasforme a éste en un lugar de trabajo compartido.

En vista de las alternativas anteriormente planteadas es necesario estructurar mecanismos de estrategias para afianzar conocimientos en el proceso enseñanza-aprendizaje, en base a la aplicación de situaciones problémicas (estudio de casos) entremezcladas con otras actividades del sistema educativo y así favorecer el aprendizaje creativo.

En el proceso de enseñanza aprendizaje se estimula el empleo de procedimiento que propicia a los alumnos el logro de la asimilación productiva de los conocimientos profesionales. Ahora bien, para utilizar adecuadamente la holística de métodos y técnicas con procedimientos metodológicos generalizados y que se produzca la asimilación productiva de los conocimientos es necesario, además, "crear las condiciones necesarias que promuevan e intensifiquen la motivación, a través de la creación de significados para los estudiantes" (Fuentes González \& Álvarez Valiente, 1998). 


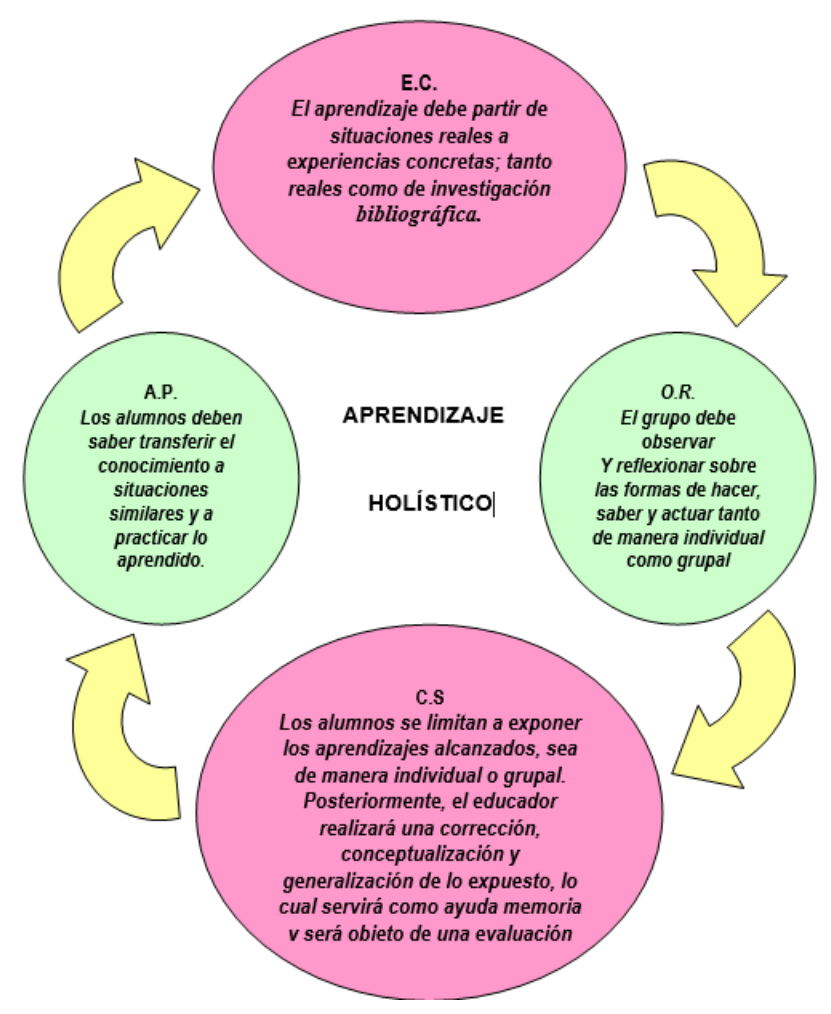

Ilustración 2. Aprendizaje holístico.

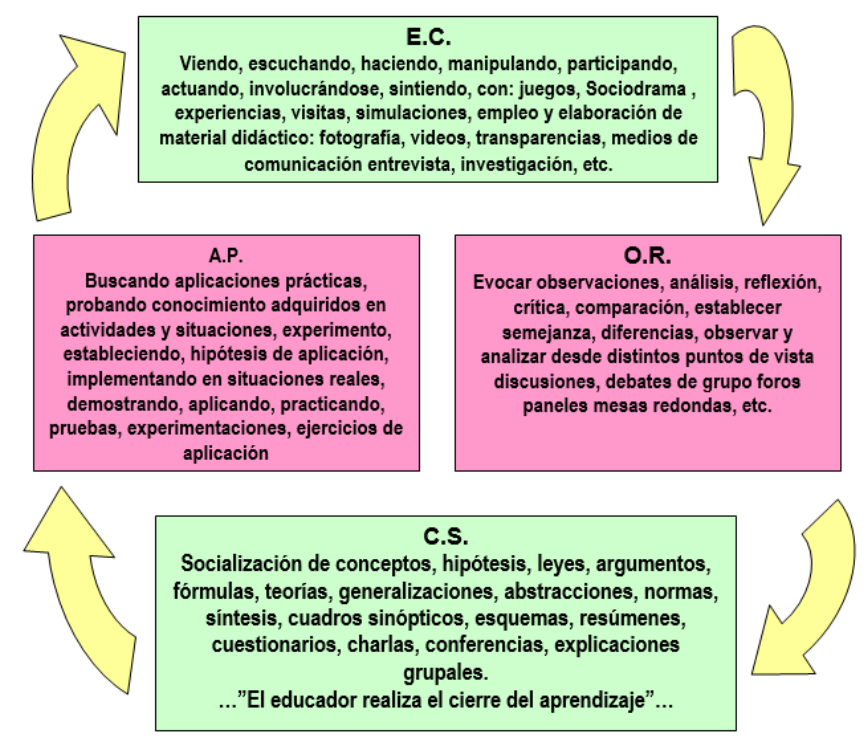

Ilustración 3. Estrategias para el proceso de aprendizaje holístico y experiencial. 


\section{MÉTODOS Y TÉCNICAS DE ENSEÑANZA}

Estos constituyen recursos necesarios de la enseñanza, son los vehículos de una realización ordenada, metódica y adecuada, de la misma. Los métodos y técnicas tienen por objeto hacer más eficiente la dirección del aprendizaje. Gracias a ellos, pueden ser elaborados los conocimientos y habilidades e incorporados con actitudes que la Universidad pretende proporcionar a sus estudiantes.

Existen muchos métodos de enseñanza pero entre los más recientes tratados por Picardo Joao, (1998), que son de gran interés para ser aplicados en centros de estudios a nivel superior. Los métodos activos de Enseñanza profesional son las formas y procedimientos que sitúan al estudiante en una posición activa, al incrementar su participación en el proceso pedagógico profesional, y ofrecerle a través de esa participación activa, las vías para la adquisición de conocimientos y el desarrollo de habilidades generalizadoras, que los capaciten para enfrentar exitosamente futuras problemáticas en el campo profesional para el cual se preparan, y para solucionar los proyectos y tareas planteadas por el docente.

El método de enseñanza es la forma particular que tiene el docente de organizar la enseñanza según Latorre y Seco del Pozo, (2013). Ejemplos de métodos de enseñanza son el método activo, pasivo, deductivo, inductivo, de resolución de problemas, etc. El estilo de aprendizaje es el camino que sigue el estudiante para aprender. Cada estudiante, con sus diferencias individuales, tiene un estilo peculiar de aprender, es decir, una manera concreta de recorrer el camino del aprendizaje. Ejemplos de estilos de aprendizaje son el estilo activo, reflexivo, pragmático, visual, etc.

\section{LA CALIDAD EN EL PROCESO DE APRENDIZAJE}

El concepto de calidad se entiende de acuerdo a la definición dada por la UNESCO, (1998), cuando plantea que "la calidad de la educación superior es un concepto multidimensional que debería comprender todas sus funciones y actividades".

De acuerdo con Pérez, Álvarez, Pérez, y Guerra, (2007), se identifican las siguientes dimensiones principales en relación a la calidad de la educación:

La calidad entendida como eficacia. Una educación de calidad es aquella que logra que los alumnos realmente aprendan lo que se supone deben aprender - aquello que está establecido en los planes y programas curriculares- al cabo de determinados ciclos o niveles. Esta dimensión del concepto pone en primer plano los resultados de aprendizaje efectivamente alcanzados por la acción educativa.

La calidad de lo que se aprende en el sistema y su relevancia en términos individuales y sociales. En este sentido, una educación de calidad es aquella cuyos contenidos responden adecuadamente a lo que el individuo necesita para desarrollarse como persona -intelectual, afectiva, moral y físicamente-, y para desempeñarse adecuadamente en los diversos 
ámbitos de la sociedad -el político, el económico, el social. Esta dimensión pone en primer plano los fines atribuidos a la acción educativa y su concreción en los diseños y contenidos curriculares.

La calidad de los procesos y medios que el sistema brinda a los alumnos para el desarrollo de su experiencia educativa. Desde esta perspectiva, una educación de calidad es aquella que ofrece a los estudiantes un adecuado contexto físico para el aprendizaje, un cuerpo docente adecuadamente preparado para la tarea de enseñar, buenos materiales de estudio y de trabajo, estrategias didácticas adecuadas, etc. Esta dimensión del concepto pone en primer plano el análisis de los medios empleados en la acción educativa. Para Chirinos Morelo \& Padron Anez, (2010), más allá de los contenidos, durante la práctica docente el término eficiencia juega un papel importante en el logro de la calidad educativa, porque tiene que ver con la acción de hacer correctamente las tareas, pasando a ser la columna vertebral de la cual dependerá el logro de los objetivos. De allí que el objetivo de esta investigación fue describir, analizar e interpretar los hechos y sucesos in situ para lograr una comprensión del perfil del docente eficiente. A través de los resultados mostraron una eficiencia centrada en la maximización de los recursos y el desarrollo de las potencialidades del alumno. Así mismo, emergieron categorías que contribuyeron a visualizar la eficiencia docente desde la perspectiva de un grupo de actores en un marco institucional. (pp. 481492).

6. HACIA UNA NUEVA VISIÓN DEL MUNDO. LA VISIÓN HOLISTA

Surge la imperiosa necesidad de buscar alternativas que ayuden a producir conocimientos, estos indudablemente deben tener la característica de científicos, comprobado está que la visión racionalista y su método no es el camino más adecuado para la producción científica, eso se debe a que deja fuera el estudio de la conciencia en el proceso mismo de producción de ciencia. "La ciencia es posible por la existencia de la conciencia, si no existiera la conciencia no se podría producir ciencia. Desde la visión holista, la nueva ciencia construye nuevos conceptos para estudiar las experiencias de los organismos con conciencia. Esto incluye, de manera central, la experiencia espiritual humana. La nueva ciencia con conciencia percibe el universo no como un conjunto de objetos, sino como una comunidad de sujetos» Gallegos R., (1999, p.11), citado por (Espino de Lara, s/f, p.5). Indiscutiblemente, debemos abordar en el ámbito educativo el asunto de la visión holista, ya que debe ser vista como una alternativa para superar los paradigmas dogmáticos y cientificistas. Reitero, la visión holista rompe con el paradigma científico mecanicista al basarse en nuevos principios de comprensión de la realidad:

- Unidad.

- Totalidad.

- Desarrollo cualitativo.

- Transdisciplinariedad. 
- Espiritualidad. Aprendizaje (Idem, p.17-18).

El sociólogo alemán Jurgen Habermas habla acerca de cómo la racionalidad instrumental impide al individuo tener una visión holística de la realidad, en consecuencia se encuentra condicionado e impedido para contar con dicha visión. En este sentido "considera insuficiente interpretar a la modernidad como un proceso de racionalización, cuando ésta última se remite exclusivamente a la razón funcional y deja de lado todas las formas de acción social-acción comunicativa» (Yurén, 1995, p.61), citado por (Espino de Lara, s/f, p.6).

El término "autenticidad», característica del individuo que puede llegar a constituir un problema de importancia en nuestra sociedad, puede ser dimensionado desde varios puntos de vista, sin embargo, casi todos podrían coincidir en considerarlo un factor que favorece al individualismo. "La idea fundamental es que hay un "ideal moral", un ideal al que todos deberíamos aspirar, que consiste en que la sociedad debe no sólo permitir, sino promover la realización de cada individuo, el despliegue de las potencialidades de cada persona para que haga, pero sobre todo para que "sea", lo que verdaderamente desea hacer y quiere ser, de acuerdo con sus convicciones, sus intereses, y en general de acuerdo con sus características individuales» (Olivé, 1996, p.8), citado por (Espino de Lara, s/f, p.6).

\section{PRINCIPIOS DE LA EDUCACIÓN HOLISTA}

En la educación holista, aprender es un concepto que adquiere una connotación especial, difiere mucho del concepto que se tiene en la educación mecanicista, desde la educación holista, «aprender es un proceso que implica muchos niveles de la conciencia humana como el afectivo, físico, social y espiritual, rebasando por completo lo puramente cognitivo y memorístico. Aprender se convierte en proceso creativo y artístico; aprender a aprender es el propósito de la educación para el siglo XXI» (Gallegos, 1999, p. 39).

Como antecedente a la intención de abordar el estudio de este tipo de educación, un grupo de educadores holísticos internacionales se reunió en la ciudad de Chicago, Illinois, en 1990 donde «el objetivo de este planteamiento es proclamar una visión alternativa de la educación, una educación que construya una respuesta vivificante y democrática a los retos de la actualidad» (Global Alliance For Transforming Education, 1995, p.7).

La educación holista es más un arte que una tecnología. Percibe al mundo en términos de relación e integración, reconoce que toda la vida en la tierra está organizada en una vasta red de interrelaciones. Cuando los principios holísticos son aplicados a la educación la escuela empieza a funcionar como un sistema vivo, como una comunidad de aprendizaje, porque los sistemas vivos son por naturaleza comunidades de aprendizaje (Lopez, 1997), La educación holista no se reduce a ser un método educativo, se caracteriza por ser una visión integral de la educación.

"El holismo es una doctrina filosófica contemporánea que tiene su origen en los planteamientos del filósofo sudafricano Smuts (n.1926) (...)” (Briceño et al., 2010, p.74). Por qué partimos del holismo obedece a la noción de totalidad, de integralidad que nos remite 
al ser humano como una entidad multidimensional, y cuya formación debe tener tal cariz y en virtud de la cual, las diversas dimensiones se vean beneficiadas de dicho proceso formativo. "Etimológicamente el holismo representa "la práctica del todo" o "de la integralidad", su raíz holos, procede del griego y significa "todo", "íntegro", "entero", "completo", y el sufijo ismo se emplea para designar una doctrina o práctica" (Briceño et al., 2010, p.74).

En tal sentido, "la holística es definida como un fenómeno psicológico y social, enraizado en las distintas disciplinas humanas y orientada hacia la búsqueda de una cosmovisión basada en preceptos comunes al género humano" (Briceño et al., 2010, p. 74). En otras palabras, la idea de fondo del holismo, es la de la comprensión de los fenómenos desde la multidimensionalidad, pues la realidad está compuesta de una diversidad de variables que interactúan entre sí, tejiendo una urdimbre compleja que posteriormente, el ser humano a partir de sus esfuerzos cognitivos, procura deshilar a fin de comprenderla.

La fundamentación del MEH que surge de esta investigación se inspira y se fundamenta en el trabajo Educación integral: una educación holística para el siglo XXI, realizado por (Yus, 2001), y (Masías, 1995). Es así que los quehaceres del modelo de educación holística “(...) se vinculan a estrategias de aprendizaje centradas en la persona que funciona como educando, bajo el predicamento de desarrollar en él procesos cognitivos, precisados en capacidades, destrezas y habilidades, y procesos socio afectivos y culturales, sobre la base de valores y de actitudes" (Romeo Cardone, 2001, p.119).

Con miras a plantear a modo de síntesis los puntos anteriormente señalados y antes de explicitar el planteamiento del MEH, nos asimos a la propuesta de Yus (2001), quien menciona que la educación holística establece las siguientes conexiones en todas las esferas de la vida:

- Relaciones entre pensamiento lineal e intuición: metáfora, visualización.

- Relaciones entre mente y cuerpo: danza, ejercicios de concentración y relajación.

- Relaciones entre dominios de conocimiento: el lenguaje global.

- Relaciones entre el yo y la comunidad: destrezas interpersonales, destrezas de servicio comunitario y destrezas de acción social.

- Relaciones entre el yo y el yo: intereses universales de los seres humanos.

\section{DIMENSIONES DEL MODELO DE EDUCACIÓN HOLÍSTICA}

El desarrollo de las Inteligencias Múltiples es el detonante de la escalera de aprendizaje propuesto en el $\mathrm{MEH}$, en el cual se plantean tres dimensiones básicas: 1. Dimensión personal 2. Dimensión interpersonal 3. Dimensión ecológica. 
A continuación se plantea el ecológica A continuación se plantea el abordaje de las dimensiones de aprendizaje del modelo fundamentadas en la postura de Educación Holística de Yus (2001), (ver ilustración 2).

\section{Dimensión personal Dimensión interpersonal Dimensión ecológica
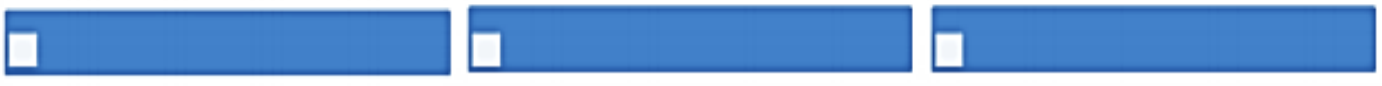 \\ Aprender a ser persona \\ Aprender el concepto de ciudadania \\ Aprender el carácter de sustentabilidad \\ $\square$ Aprender a integrar mente-cuerpo- \\ alma \\ Aprender a trabajar con los demás para el blen común \\ Aprender los vínculos interactivos con el entorno natural y social, personal \\ Aprender a desarrollar lo físico, \\ cognitivo y espiritual \\ Aprender a ser empático y tolerante con el otro \\ Aprender a desarrollar las \\ dimensiones personal y social \\ Aprender a vivir los valores ytranspersonal \\ $\square$ Aprender la ecoformación \\ Aprender la conciencia ecológica \\ Aprender a reflexionar del debate, de \\ las diferencias en ideología \\ Aprender a convivir}

Ilustración 4. Dimensiones de aprendizaje para la formación holística.

Fuente: Gluyas (2010).

La dimensión personal se enfoca en aprender a ser persona a través de una conexión entre mente y cuerpo para activar los aspectos cognitivo, físico y espiritual como parte del proceso formativo para desarrollar al máximo el potencial humano. Aquello que nos diferencia y nos hace únicos sin abandonar los planteamientos sociales y la cooperación para el bien común. Se trata de aprender a ser persona para reaprender lo que significa saber reflexionar a partir del autoconocimiento y la autogestión.

La dimensión interpersonal plantea una educación cívica y social, que promueva la reflexión y la tolerancia sobre diversos planteamientos. Fomenta la educación en valores para aprender a convivir con la base del respeto y la conciencia ciudadana.

La dimensión ecológica propone aprender el carácter de la sustentabilidad a partir de las relaciones entre todos los elementos humanos. Desde un enfoque transdisciplinar trata los derechos de la persona y del planeta, con una conciencia ecológica que va más allá de la educación ambiental para poner de manifiesto las relaciones entre la humanidad y la Tierra.

De los resultados obtenidos de una investigación realizada a estudiantes de la carrera de Computación y Redes de la Universidad Estatal del Sur de Manabí, se presenta alternativa para un trabajo docente educativo eficiente en base a una visión holística aplicando métodos y técnicas didácticas en el proceso enseñanza aprendizaje en la Educación Superior. Cabe resaltar que con un trabajo docente educativo de calidad se logra formar profesionales competitivos. 


\section{CONCLUSIONES}

Los componentes del trabajo docente en el desarrollo del proceso enseñanza aprendizaje concluyó con gran relevancia en la aplicación de métodos y técnicas didácticas para una educación con visión holística que es la imagen del cumplimiento de un aprendizaje global, donde los estudiantes aprenden eficazmente y de esta manera se logra el cambio de mentalidad con una educación más activa participativa y creativa, haciendo uso de recursos didácticos para un aprendizaje significativo.

De igual manera, es necesario implementar una guía metodológica de recursos didácticos con aplicaciones tecnológicas para el desarrollo del trabajo docente educativo dentro del proceso enseñanza aprendizaje, ya que un docente altamente preparado impartirá una educación de calidad con el uso de herramientas didácticas que fortalecen el proceso de aprendizaje.

\section{REFERENCIAS BIBLIOGRÁFICAS}

Amidon, E., y Hunter, E. (1996). Mejoramiento del aprendizaje: análisis verbal interacción en el aula. New york: Holt Rinehart y Wiston. Recuperado de: $<$ http://www.uson.mx/difusiondelacultura/revista psicumex/emag/psicumex5/ind ex.htm|\#/24/>.

Ausubel, \& Novak, J. (1995). Significado y aprendizaje significativo, Psicología educativa. México: Edit. Trillas. Recuperado de: $<$ http://mastereducacionsalva.blogspot.com/2012/02/propuesta-ensayo-finalevec.html/>.

Bigge L., M., \& Hunt. P. (1978). Bases Pedagógicas para la Educación.

Briceño , J., Cañizales, B., Rivas, Y., Lobo, H., Moreno, E., Velásquez, I., y Ruzza, I. (2010). La holística y su articulación con la generación. Revista Electrónica "Actualidades Investigativas en Educación", 15(3), 73-83. DOI: <http://www.redalyc.org/articulo.oa?id=35616720008/>.

Chirinos Morelo, N., \& Padron Anez, E. (2010). La eficiencia docente en la práctica educativa. Revista de Ciencias sociales [online] (n 3), pp. 481- 492. Recuperado el 07 de junio de 2014, de: $<$ http://www.scielo.org.ve/scielo.php?script=sci arttext\&pid=S131595182010000300009\&lng=es\&nrm=iso/>. ISSN 1315-9518.

Christian, C. (2016). Aplicativo móvil como estrategia de marketing para el impulso de la matriz productiva en el área turística. 3c Tecnología , 41- 53. 
De Leon C., I. (2013). Gestión del conocimeinto, formación docente de Educación Superior y desarrollo de Esilos de Enseñanza: interacciones e interrelaciones. Revista de Investigación, Caracas, 37, (79). Recuperado el 06 de julio de 2014, de: $<$ http://www.scielo.org.ve/scielo.php?script=sci arttext\&pid=S1010$29142013000200010 \&$ Ing=es\&nrm=iso/>.

Espino de Lara, R. (s/f). Educación Holítica. OEl-Revista Iberoamericana de Educación, 6. Recuperado de: <http://www.rieoei.org/deloslectores/330Espino.pdf/> ISSN: 16815653.

Fuentes González, H., y Álvarez Valiente, I. (1998). Dinámica del proceso docente educativo de la educación superior. CEES "Manuel F. Gran". Universidad de Oriente. Santiago de Cuba.

Gallegos, R. (1999). Educación Holista: Pedagogía del amor universal. OEl-Revista Iberoamericana de Educación, 11 . Recuperado de: $<$ http://www.rieoei.org/deloslectores/330Espino.pdf/>. ISSN: 1681-5653.

Gallegos, R. (2005). Educación holista para el siglo XXI. (Documento: Una visión integral de la educación). Fundación internacional para la educación holista. México, DF. Recuperado de: <http://recursosbiblio.url.edu.gt/tesiseortiz/2014/05/86/TaxEdgar.pdf/>.

Gallegos, R. (2005). Educación holista para el siglo XXI. (Documento: Una visión integral de la educación). Fundación internacional para la educación holista. México. México, DF. Recuperado de: <http://recursosbiblio.url.edu.gt/tesiseortiz/2014/05/86/TaxEdgar.pdf/> <www.ramongallegos.com/RamonGallegosEducacion/ $>$.

García Alvarez, N. d., Trujillo Alonso, B., y Perez Duarte, W. (2010). Propuesta metodológica para mejorar el proceso de enseñanza aprendizaje de la asignatura Calidad del agua. Rev Cie Téc Agr, 19(1). Recuperado el 08 de junio de 2014 de $<$ http://scielo.sld.cu/scielo.php?script=sci arttext\&pid=S207100542010000100016\&lng=es\&nrm=iso/>.

Global Alliance For Transforming Education. (1995). En: Cuadernos de formación de investigadores. México, Universidad Autónoma Chapingo. OEl-Revista Iberoamericana de Educación. Recuperado de: $<$ http://www.rieoei.org/deloslectores/330Espino.pdf/>. ISSN: 1681-5653.

Latorre Ariño, M., \& Seco del Pozo, C.J. (2013). Metodología Estrategias y técnicas metodológicas (1a ed.), (M. C.-L.-L. Perú, Ed.) Lima Perú: visionpcperu. Recuperado de: $<$ https://es.scribd.com/doc/207835347/UMCH-Metodologia/>.

Lopez, R. (1997). Complejidad y educación. Revista Española de Pedagogía 206: 103-112. Recuperado de: <http://araguaney.me.gob.ve/?page id=99/>. 
Marcuse, H. (2001). El hombre unidimensional. Revista Electrónica "Actualidades Investigativas en Educación". Recuperado de: $<$ http://revistas.ucr.ac.cr/index.php/aie/article/view/20654/21074/>.

Masías, J. (1995). La ética en la universidad. Orientaciones básicas. Revista Electrónica "Actualidades Investigativas en Educación". Recuperado de: <http://revistas.ucr.ac.cr/index.php/aie/article/view/20654/21074/>.

Medina , A., Sevillano , M., \& de la Torre , S. (2009). Una universidad para el siglo XXI. Espacio Europeo de Enseñanza Superior (EEES). Una mirada transdisciplinar, ecoformadora e intercultural. Revista Electrónica "Actualidades Investigativas en Educación". Recuperado de: $<$ http://revistas.ucr.ac.cr/index.php/aie/article/view/20654/21074/>.

Montes de Oca, R. N., \& Machado Ramírez, E. F. (2011). Estrategias docentes y métodos de enseñanza aprendizaje en la Educación Superior. Rev. Hum med [online]. 11(3): 475-488. Recuperado el 08 de junio de 2014, de: $<$ http://scielo.sld.cu/scielo.php?script=sci arttext\&pid=S1727$81202011000300005 \& \operatorname{lng}=\mathrm{es} />$.

Olivé, L. (1996). «Racionalidad y autenticidad: desafíos para la educación», en: IBARRA, Manuel (coord.): La. OEI-Revista Iberoamericana de Educación), 8. Recuperado de: $<$ http://www.rieoei.org/deloslectores/330Espino.pdf/>. ISSN: 1681-5653.

Pérez Morales, J. I., Álvarez Valdivia, I. M., Pérez Cabaní, M. L., \& Guerra Rubio, L. M. (2007). La evaluación como instrumento de mejora de la calidad del aprendizaje, propuesta de intervenión psicopedagógica para el aprednizaje del idioma inglés. Tesis doctoral. Universidad de Girona Universidad central Marta Abreu de las Villas .

Picardo Joao, Ó. (1998 Noviembre- Diciembre). Ideología y Desideologización: Aproximación al concepto. En Theorethicos. Revista electrónica de la Universidad Francisco Gavidia, 1(6). Recuperado de: <http://www.ufg.edu.sv/ufg/theorethikos/Noviembre98/ideologia.html/>.

Romeo Cardone, J. (2001). Los objetivos fundamentales transversales en busca de un currículo holístico. Estudios Pedagógicos,. Revista Electrónica "Actualidades Investigativas en Educación" (27), 119-130. DOI: $<$ http://www.redalyc.org/articulo.oa?id=173513844009/>.

Sierra Eupierre, Y., Castellano mesa, E., \& García Pérez, Y. (2013). Preparación de los docentes para dirigir el proceso de enseñanza aprendizaje utilizando niveles de asimilación. EDUMECENTRO [online] 5(3): 95-107. Recuperado el 06 de julio de 2014, de: $\quad$ http://scielo.sld.cu/scielo.php?script=sci arttext\&pid=S207728742013000300007\&lng=es/>.

UNESCO. (1998). La educación superior en el siglo XXI Visión y acción. París. 
Vera Bachmann, D., Osses, S., \& Schiefelbein Fuenzalida, E. (2012). Las creencias de los profesores rurles: una tarea pendiente para la investigación educativa. Estudios pedagógicos. Estudios Pedagógicos (Valdivia), 297-310. Recuperado el 06 de julio de 2014, de: <http://www.scielo.cl/scielo.php?script=sci arttext\&pid=S0718-

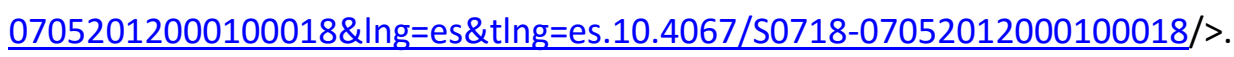

Yurén, M. (1995). Eticidad, valores sociales y educación. (U. P. Nacional., Ed.) OEI-Revista Iberoamericana de Educación, 61. Recuperado de: $<$ http://www.rieoei.org/deloslectores/330Espino.pdf/>. ISSN: 1681-5653.

Yus, R. (2001). Educación integral: una educación holística para el siglo XXI. Revista Electrónica "Actualidades Investigativas en Educación". Recuperado de: $<$ http://revistas.ucr.ac.cr/index.php/aie/article/view/20654/21074/>.

Zabalza Beraza, M. A. (2004). Guía para la planificación didáctica de la docnecia universitaria en el marco del EEES (guía de guías) documento de trabajo. Universidad Santiago de Compostela. 